QUARTERLY OF APPLIED MATHEMATICS

VOLUME LXIX, NUMBER 2

JUNE 2011, PAGES 205-225

S 0033-569X(2011)01193-7

Article electronically published on March 3, 2011

\title{
TRANSIENT TEMPERATURE RESPONSE OF SPHERICAL BODIES
}

\author{
BY \\ BEJOY K. CHOUDHURY \\ Lockheed Martin Space Systems, Sunnyvale, California \\ In memory of late Professor I. M. Cohen [1937-2007], whose lifelong dedication to teaching and \\ research has inspired, and continues to inspire
}

\begin{abstract}
Fundamental temperature solutions in closed form of composite spherical bodies are given for mixed and time-dependent boundary conditions. Solid and hollow spherical bodies are included as further examples. The solution requires calculating the roots of certain transcendental equations. A method is developed to find the roots rapidly. As a practical application, the two-layer composite solution is used to determine the available fuel mass of an orbiting spacecraft.
\end{abstract}

1. Introduction. The study of the radiation cooling of spherical bodies has a rich history. It has the distinction of being the first natural science approach to discredit James Ussher's young earth account and to refute Lyell's uniformitarian theory. Kelvin (William Thomson), who was profoundly influenced by Fourier's Théorie Analytique de la Chaleur (1822), was the first to calculate earth's cooling rate from a consideration of the distribution of heat within the earth (1862). His estimation of the earth as one hundred million years and not older, however, ushered in the protracted debate that was not settled until Russell in 1921, and Rutherford in 1929, calculated the earth's age from the radioactive decay of uranium.

Radiation cooling continued to draw interest well into the twentieth century. The 1904 Cambridge Mathematical Tripos II examination set one composite spherical heat conduction problem and asked candidates to explain the bearing of a numerical solution on the age of the earth. Carslaw [5, also Bromwich 4, reports that the solution given in the paper was in error because an incorrect interface condition $\kappa_{1} \partial\left(u_{1} r\right) / \partial r=\kappa_{2} \partial\left(u_{2} r\right) / \partial r$ was used (cf. (2.4) below).

Fourier's generalized heat equation and Navier's generalized strain-displacement equations become uncoupled under certain restrictions. The conditions under which such uncoupling is valid are discussed fully in [3, [10]. For composite spheres, uncoupled stress

Received May 1, 2009.

2000 Mathematics Subject Classification. Primary 80A20, 44A10.

Key words and phrases. Heat conduction, diffusion, radiation, Laplace transform.

E-mail address: bejoy.choudhury@lmco.com

(C)2011 Brown University Reverts to public domain 28 years from publication 
and displacement are expressed in terms of $\vartheta=T-T_{\text {ref }}$, where $T_{\text {ref }}$ is the temperature of the body in an unconstrained reference state [3, p. 301], [17, p. 297]. Hata [14 has investigated elastic and creep deformation in the sphere due to the sudden cooling in a hot bath. Gladwell and Barber [11] use the temperature solution for the half-space under radiation boundary conditions to solve thermoelastic contact problems. It appears that had the more general analytical solutions been available, the authors might have used them to advantage. Whether or not the physics of thermomechanic interaction is sufficiently far advanced, and authors differ on this [10, viii], uncoupled temperature solutions continue to find practical uses.

Most problems of interest involve boundary conditions of the second and third kind. In this paper we study the response of composite spherical bodies with a radiation condition and a time-varying heat flux at the surface. Mixed boundary conditions are also considered. Composite solutions have diverse use, but as J. C. Jaeger has remarked the work is tedious (Phil. Mag. (1944) p. 172). In the present case the extra parameters involved make general solutions difficult and are not available [9]. The difficulty is due to the algebraic nature of such problems, and the fact that certain transcendental equations must be solved. Explicit formulations of the transient heat conduction problem in polar coordinates with multiple annular layers are given in [15], 21]. The resulting equations were solved numerically. In this paper we shall concentrate on the two-layer spherical body, but the theory can be extended to multi-layers. Transient solutions are presented in $\S \S 2-3$. In $\S 4$ we present a rapid method of determining complete roots of the eigenvalue equations. The method and the solutions should apply to a wide variety of heat and mass transfer, and thermoelasticity problems [9], 18, [7, [3], 17]. Extension to multi-dimensional problems using weighted residual methods, such as the Galerkin method, is possible by taking a combination of basis functions suggested by the solutions presented, and associated Legendre functions as needed.

In $\S 5$, a composite sphere solution is used to determine the residual fuel mass of an orbiting spacecraft. Currently spacecraft do not have the means to collect and relay the status of their fuel. Yet a demonstrated availability of even one $\mathrm{kg}$ of fuel could extend a satellite's life by a year. Typically, tanks are assumed to be depleted when disturbance torques cannot be suppressed or when the spacecraft's attitude will no longer be maintained. This makes the analytical determination a valuable new tool in the planning and operation of aging spacecraft.

2. General formulation. We consider a composite spherical body with two regions of dissimilar materials. Region 1 is the core $0 \leq r<a$ with $T_{1}, \rho_{1}, c_{1}, \kappa_{1}$ as its temperature, density, specific heat, and thermal conductivity. Region 2 is the outer skin $a<r \leq b$ with $T_{2}, \rho_{2}, c_{2}, \kappa_{2}$ for the same. We assume the temperature to vary only in the radial direction. Thermodynamic and transport properties are assumed constant for the temperature range of our interest. The sphere exchanges heat by radiation to its surroundings at $T_{\infty}$ and receives heat flux $q_{b}$ on the surface at $r=b$. The effective emissivity of the external surface is $\epsilon_{2}$, and $\sigma$ is the Stefan-Boltzmann constant. Initial temperatures are functions of $r$. The surface heat flux or the sink temperature, but not 
both, can be a function of time. The solution involves considerable algebra. To save space we shall omit many intermediate steps.

2.1. Composite spherical body with radiation and heat flux at the surface. We solve the Fourier differential equations (DEs)

$$
\begin{array}{ll}
\frac{\partial T_{1}}{\partial t}=s_{1}\left(\frac{\partial^{2} T_{1}}{\partial r^{2}}+\frac{2}{r} \frac{\partial T_{1}}{\partial r}\right) & (0 \leq r<a), \\
\frac{\partial T_{2}}{\partial t}=s_{2}\left(\frac{\partial^{2} T_{2}}{\partial r^{2}}+\frac{2}{r} \frac{\partial T_{2}}{\partial r}\right) & (a<r<b),
\end{array}
$$

subject to the boundary conditions (BCs)

$$
\begin{array}{rlrl}
T_{1} & =T_{2}, & r=a, \\
-\kappa_{1} \frac{\partial T_{1}}{\partial r}=-\kappa_{2} \frac{\partial T_{2}}{\partial r}, & r=a, \\
-\kappa_{2} \frac{\partial T_{2}}{\partial r}=\sigma \epsilon_{2}\left(T_{2}^{4}-T_{\infty}^{4}\right)-q_{b}, & r=b, \\
T_{1}=f_{1}(r), t=0 & (0 \leq r \leq a) ; T_{2}=f_{2}(r), t=0 & (a \leq r \leq b) .
\end{array}
$$

$T_{1}$ is finite at $r=0$. Here $s_{1}=k_{1} /\left(\rho_{1} c_{1}\right), s_{2}=k_{2} /\left(\rho_{2} c_{2}\right)$ are thermal diffusivities of the core and the outer skin. Without the loss of generality we shall neglect thermal resistance at the interface; cf. (2.4). The first term on the right-hand side of (2.5) will be linearized as $\sigma \epsilon_{2}\left(T_{2}^{4}-T_{\infty}^{4}\right)=h_{2}\left(T_{2}-T_{\infty}\right)$, where $h_{2}=4 \sigma \epsilon_{2} T_{\infty}^{3}+O\left[\left(T_{2}-T_{\infty}\right) / T_{\infty}\right]$. If the requirement $\left|\left(T_{2}-T_{\infty}\right) / T_{\infty}\right| \ll 1$ cannot be met, then linear solutions are still useful, but it will be necessary to iterate on the nonlinearity. This remark applies to all problems discussed in this paper. In $\S 5$ we discuss a problem where the linearization condition is satisfied.

To simplify the DEs (2.1)-(2.2) let $u_{i}=r T_{i}$, and then use $u_{i}=a_{i}+b_{i} r+v_{i}, i=1,2$ to homogenize the BCs. For $T_{1}$ to be finite at $r=0$ we set $a_{1}=0$. Therefore

$$
a_{1}=a_{2}=0, \quad b_{1}=b_{2}=T_{\infty}+\frac{q_{b}}{h_{2}} .
$$

The transformed equations will not be shown.

2.2. The subsidiary equations. Next we take the Laplace transform of the equations

$$
\bar{v}_{i}(r)=\int_{0}^{\infty} e^{-p t} v_{i}(r, t) d t \quad(\operatorname{Re} p>0) .
$$


With $q_{1}=\sqrt{\left(p / s_{1}\right)}, q_{2}=\sqrt{\left(p / s_{2}\right)}$, and $B i=h_{2} b / \kappa_{2}$ a dimensionless Biot number, the subsidiary equations are

$$
\begin{aligned}
\frac{d^{2} \bar{v}_{1}}{d r^{2}}-q_{1}^{2} \bar{v}_{1} & =\left(b_{1}-f_{1}\right) \frac{r}{s_{1}} & & (0 \leq r<a), \\
\frac{d^{2} \bar{v}_{2}}{d r^{2}}-q_{2}^{2} \bar{v}_{2} & =\left(b_{2}-f_{2}\right) \frac{r}{s_{2}} & & (a<r<b), \\
\bar{v}_{1} & =\bar{v}_{2}, & r & =a, \\
-\kappa_{1}\left(a \frac{d \bar{v}_{1}}{d r}-\bar{v}_{1}\right) & =-\kappa_{2}\left(a \frac{d \bar{v}_{2}}{d r}-\bar{v}_{2}\right), & r & =a, \\
-b \frac{d \bar{v}_{2}}{d r} & =(B i-1) \bar{v}_{2}, & r & =b .
\end{aligned}
$$

If the initial temperature is constant or a polynomial, then the particular integral will also be a polynomial. If we treat $f_{1}, f_{2}$ as arbitrary functions and if the boundary conditions are of the general kind, then due to the nonhomogeneity the solution and its inversion will not be simple. If there is no ambiguity, in this paper trigonometric expressions such as $\sin [(m+1) \theta]$, $\sin (q r)$ will be written as $\sin (m+1) \theta, \sin q r$. In keeping with Carslaw and Jaeger [7, p. 351], we introduce the auxiliary functions

$$
\left.\begin{array}{l}
\varphi_{i}(r)=q_{i} a \cosh q_{i} r-\sinh q_{i} r, \\
\psi_{i}(r)=q_{i} a \cosh q_{i}(r-a)+\sinh q_{i}(r-a), \\
\chi_{i}(r)=\int^{r} r^{\prime}\left[b_{i}-f_{i}\left(r^{\prime}\right)\right] \sinh q_{i}\left(r-r^{\prime}\right) d r^{\prime}
\end{array}\right\}
$$

where $i=1,2$. The last one is new. Here $\chi_{i}(r)$ is an indefinite integral. The upper limit is a reminder that the function is evaluated at $r$. The notation will help us write expressions in a compact manner; see e.g. (2.28). This requires some explanation.

Using variation of parameters, a particular solution is obtained in the usual manner. Thus, the solution of the DE (2.8) is written as

$$
\bar{v}_{2}=C \cosh q_{2} r+D \sinh q_{2} r+\frac{1}{q_{2} s_{2}} \int^{r} r^{\prime}\left(b_{2}-f_{2}\right) \sinh q_{2}\left(r-r^{\prime}\right) d r^{\prime},
$$

where $C, D$ are constants. Since any indefinite integral may be expressed as a one-sided definite integral, Hardy [13, p. 315], we can rewrite this as

$$
\bar{v}_{2}=C^{\prime} \cosh q_{2} r+D^{\prime} \sinh q_{2} r+\frac{1}{q_{2} s_{2}} \int_{a}^{r} r^{\prime}\left(b_{2}-f_{2}\right) \sinh q_{2}\left(r-r^{\prime}\right) d r^{\prime},
$$

where $C^{\prime}, D^{\prime}$ are some other constants. The choice of an upper or lower definite limit is completely arbitrary. When regard is paid to the differences, particularly in taking derivatives, both will yield identical results. The choice of $\chi_{i}$ may simplify some algebra (cf. $§ 2.5)$. With this the solution to the DEs $(2.7)-(2.8)$ is

$$
\left.\begin{array}{l}
\bar{v}_{1}=B \sinh q_{1} r+\frac{1}{q_{1} s_{1}} \chi_{1}(r), \\
\bar{v}_{2}=C \cosh q_{2} r+D \sinh q_{2} r+\frac{1}{q_{2} s_{2}} \chi_{2}(r) .
\end{array}\right\}
$$


To satisfy the BCs (2.9)-(2.11), the coefficients $B, C, D$ must satisfy

$$
\left[\begin{array}{ccc}
\sinh q_{1} a & -\cosh q_{2} a & -\sinh q_{2} a \\
\kappa_{1} \varphi_{1}(a) & -\kappa_{2}\left(q_{2} a \sinh q_{2} a-\cosh q_{2} a\right) & -\kappa_{2} \varphi_{2}(a) \\
0 & q_{2} b \sinh q_{2} b+B i^{\prime} \cosh q_{2} b & q_{2} b \cosh q_{2} b+B i^{\prime} \sinh q_{2} b
\end{array}\right]\left\{\begin{array}{c}
B \\
C \\
D
\end{array}\right\}=\frac{1}{p}\left\{\begin{array}{c}
R_{1} \\
R_{2} \\
-R_{3}
\end{array}\right\}
$$

Here for convenience we have defined $B i^{\prime}=B i-1$, and $R_{1}, R_{2}, R_{3}$ as

$$
\left.\begin{array}{l}
R_{1}=q_{2} \chi_{2}(a)-q_{1} \chi_{1}(a) \\
R_{2}=\kappa_{2} q_{2}\left[a \chi_{2}^{\prime}(a)-\chi_{2}(a)\right]-\kappa_{1} q_{1}\left[a \chi_{1}^{\prime}(a)-\chi_{1}(a)\right], \\
R_{3}=q_{2}\left[(B i-1) \chi_{2}(b)+b \chi_{2}^{\prime}(b)\right] .
\end{array}\right\}
$$

The determinant of the left-hand-side matrix is

$$
\begin{aligned}
\Delta=\kappa_{1}\left[q_{2} b \cosh q_{2}(b-a)\right. & \left.+(B i-1) \sinh q_{2}(b-a)\right] \varphi_{1}(a) \\
& +\kappa_{2}\left[(B i-1) \psi_{2}(b)+b \psi_{2}^{\prime}(b)\right] \sinh q_{1} a .
\end{aligned}
$$

Also, $B=\Delta_{1}^{\prime} /(p \Delta), C=\Delta_{2}^{\prime} /(p \Delta), D=\Delta_{3}^{\prime} /(p \Delta)$, where $\Delta_{1}^{\prime}, \Delta_{2}^{\prime}, \Delta_{3}^{\prime}$ are some algebraic expressions. It is clear that the recovery of $v_{1}, v_{2}$ with the aid of tables of inverse Laplace transforms will not be possible.

2.3. The inversion of $\bar{v}_{1}, \bar{v}_{2}$. In the complex domain we shall use the variables $\lambda$, $\mu_{i}$ instead of $p, q_{i}$. Let $\mu_{1}=\sqrt{\left(\lambda / s_{1}\right)}, \mu_{2}=\sqrt{\left(\lambda / s_{2}\right)}, K=\sqrt{\left(s_{1} / s_{2}\right)}, \sigma=K \kappa_{2} / \kappa_{1}$, $m=K[(b / a)-1]$. The definition of $\sigma$ here differs from Carslaw and Jaeger's use of $\sigma=\kappa_{1} /\left(K \kappa_{2}\right)$ in spherical and $\sigma=K \kappa_{2} / \kappa_{1}$ in rectangular problems [7. By the Inversion Theorem,

$$
\left.\begin{array}{l}
v_{1}(r, t)=\frac{1}{2 \pi i} \int_{\gamma-i \infty}^{\gamma+i \infty} e^{\lambda t}\left[\frac{\Delta_{1}^{\prime}}{\Delta} \sinh \mu_{1} r+\frac{\lambda}{\mu_{1} s_{1}} \chi_{1}(r)\right] \frac{d \lambda}{\lambda}, \\
v_{2}(r, t)=\frac{1}{2 \pi i} \int_{\gamma-i \infty}^{\gamma+i \infty} e^{\lambda t}\left[\frac{\Delta_{2}^{\prime} \cosh \mu_{2} r+\Delta_{3}^{\prime} \sinh \mu_{2} r}{\Delta}+\frac{\lambda}{\mu_{2} s_{2}} \chi_{2}(r)\right] \frac{d \lambda}{\lambda} .
\end{array}\right\}
$$

For contour integration of this, $\gamma$ may be taken as large as we wish. We first dispose of the second terms. Each term is a regular function of complex variables with no poles or singularities, and therefore by Cauchy's theorem the second terms do not contribute to the integrals. Let $\mu_{n}=-i \beta_{n} / a$. The remaining integrands are single-valued functions, the only singularities being the poles at $\lambda_{n}=-\left(s_{1} / a^{2}\right) \beta_{n}^{2} \leq 0, n=0,1,2, \ldots$. The poles are real and simple and are given by $\Delta=0$, or

$$
\begin{aligned}
-\kappa_{1}[ & \left.\frac{K b}{a} \beta \cos m \beta+(B i-1) \sin m \beta\right](\beta \cos \beta-\sin \beta) \\
& -\kappa_{2}\left\{\frac{K b}{a} \beta[\cos m \beta-K \beta \sin m \beta]+(B i-1)[K \beta \cos m \beta+\sin m \beta]\right\} \sin \beta=0 .
\end{aligned}
$$




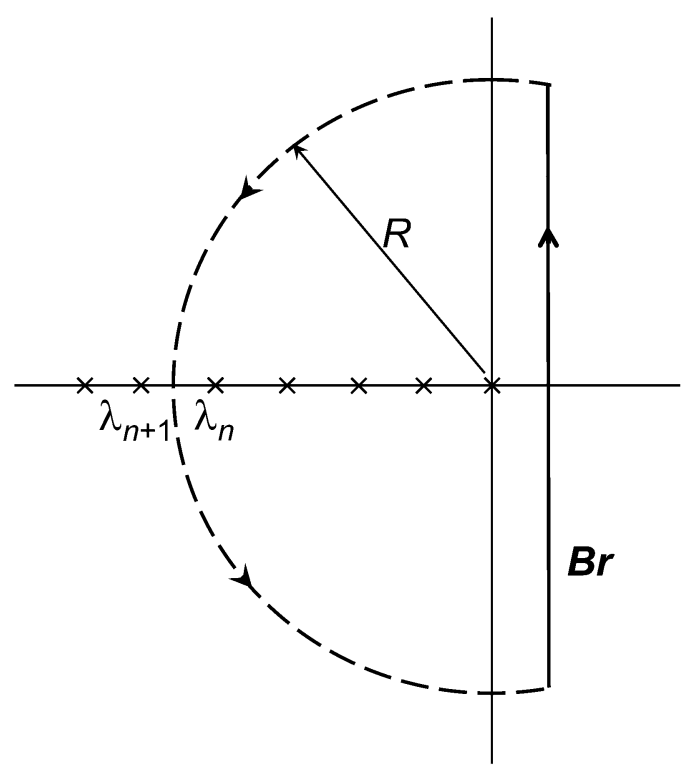

Fig. 1. Bromwich contour for integrals (2.18), (2.19). An infinite number of poles lie on the negative real axis. The poles are all simple and the integrands have no branch points.

The singularities of the integrands in (2.18)-(2.19) lie on the left-hand side of a Bromwich path $\boldsymbol{B r}$, and we take the Bromwich contour shown in Fig. 1. The contour crosses the negative real axis between the $n$th and $(n+1)$ th poles. In $\S 2.4$ we examine the contribution to the integral from the half-circle and show that it is zero. Then by the residue theorem the integral is $2 \pi i$ times the sum of the residues at the poles. Finally, we deform the contour into a line integral and take $n \rightarrow \infty$. The last step produces the desired solution in infinite series.

In the present problem it will be convenient to reduce the integrands to types $w=$ $f(z) / g(z)$ or $w=f(z) /\left[z^{s} g(z)\right], s>0$. Here the numerator and the denominator have no common zeros and $g(z) \equiv \prod_{n=1}^{\infty}\left(z-\lambda_{n}\right), \lambda_{n}>0$. We can always write in this way even if $g(z)$ is not easily factorizable. If $s=1$, we have [16]

$$
\frac{1}{2 \pi i} \int_{C} \frac{f(z)}{z g(z)} d z=\frac{f(0)}{g(0)}+\left.\sum_{n=1}^{\infty} \frac{f(z)}{z g^{\prime}(z)}\right|_{z=\lambda_{n}}
$$

The poles are the roots of (2.20), i.e.,

$$
\begin{aligned}
\sigma\left[B i+\left(1-\frac{\kappa_{1}}{\kappa_{2}}\right) \frac{b}{a}\right. & -1] \cot m \beta+(B i-1) \cot \beta \\
& +\frac{K b}{a} \beta(\cot \beta \cot m \beta-\sigma)=-(B i-1) \frac{\zeta}{\beta} .
\end{aligned}
$$


Before proceeding further we mention two special cases:

$$
\begin{array}{ll}
\sigma \cot m \beta+\cot \beta+\frac{\zeta}{\beta}=0 & (B i \rightarrow \infty) \\
\sigma \tan m \beta-\cot \beta-\frac{\zeta}{\beta}=0 & (B i=1)
\end{array}
$$

Here $\zeta=\left(\kappa_{2} / \kappa_{1}\right)-1$. The method of solution of equations $(2.22)-(2.24)$ is deferred until $\S 4.4$. The residues at the pole at $\lambda=0$ cancel out the nonhomogeneous terms of (2.7), (2.8). Hence for $\beta_{n}>0$, the temperature solution is

$$
\left.\begin{array}{c}
T_{1}(r, t)=b_{1}-\frac{2}{\kappa_{1} r} \sum_{n=1}^{\infty} \frac{\sin \beta_{n} \sin m \beta_{n}}{\Phi\left(\beta_{n}\right)}\left(i \Delta_{1}^{\prime}\right)_{\lambda=\lambda_{n}} \sin \left(\frac{\beta_{n} r}{a}\right) \exp \left(-F o \beta_{n}^{2}\right), \\
T_{2}(r, t)=b_{2}-\frac{2}{\kappa_{1} r} \sum_{n=1}^{\infty} \frac{\sin \beta_{n} \sin m \beta_{n}}{\Phi\left(\beta_{n}\right)}\left[\left(\Delta_{2}^{\prime}\right)_{\lambda=\lambda_{n}} \cos \left(\frac{K \beta_{n} r}{a}\right)\right. \\
\left.-\left(i \Delta_{3}^{\prime}\right)_{\lambda=\lambda_{n}} \sin \left(\frac{K \beta_{n} r}{a}\right)\right] \exp \left(-F_{o} \beta_{n}^{2}\right) .
\end{array}\right\}
$$

Here $F_{O}=s_{1} t / a^{2}$ is a dimensionless Fourier number and

$$
\begin{aligned}
\Phi\left(\beta_{n}\right)= & \sigma\left[B i+\left(1-\frac{\kappa_{1}}{\kappa_{2}}\right) \frac{b}{a}-1\right] m \beta_{n}^{2} \sin ^{2} \beta_{n}+(B i-1) \beta_{n}^{2} \sin ^{2} m \beta_{n} \\
& +\left[\frac{\sigma K b}{a} \beta_{n}^{2}+(B i-1) \zeta\right] \sin ^{2} \beta_{n} \sin ^{2} m \beta_{n} \\
& +\frac{K b}{2 a} \beta_{n}^{2}\left[\beta_{n} \sin 2 m \beta_{n}+m \beta_{n} \sin 2 \beta_{n}-\frac{1}{2} \sin 2 \beta_{n} \sin 2 m \beta_{n}\right],
\end{aligned}
$$

and $b_{1}=b_{2}=T_{\infty}+q_{b} / h_{2}, i=\sqrt{-1}$. Both $i \Delta_{1}^{\prime}, i \Delta_{3}^{\prime}$ are real. The solution is valid for all $B i$ except possibly when $B i=0$ but $q_{b} \neq 0$. For, if the surface is insulated, the body may be receiving more heat than it can absorb and therefore the problem may not possess a steady state solution. This case is fully discussed by Boley and Weiner [3, p. 201] and in 8. We note in passing that Tauberian theorems, Heaviside's method, and Watson's lemma are available for 'small' and 'large' times, and that transient solutions subsume steady state solutions and thus steady state solutions are not discussed separately.

2.4. To show that the contribution to the integrals from the semicircle is zero as its radius $R \rightarrow \infty$. To complete the solution it will be necessary to show that the contribution to the integrals from the semicircle path, Fig. 1, is zero. To demonstrate this we recall a theorem due to Carslaw and Jaeger [6, p. 398] which states: on the contour path $\Gamma$ where $\lambda=R e^{i \theta},-\pi \leq \theta \leq \pi, R>R_{0}$, if $R_{0}, C, k>\frac{1}{2}$ are constants independent of $\theta$ and $\xi$ such that $f(\lambda, \xi)<C R^{-k} \exp \left(-\xi \sqrt{ } R \cos \frac{1}{2} \theta\right)$, then

$$
\lim _{R \rightarrow \infty} \int_{\Gamma} e^{\lambda t} f(\lambda, \xi) \frac{d \lambda}{\lambda}=0
$$

provided that the parameter $\xi \geq 0, t>0$. If $\xi>0$, then the statement holds also when $t=0$. 
Let $\lambda=R e^{i \theta}$, where $R=s_{1} \bar{\beta}^{2}, \bar{\beta}=\left(\beta_{n}+\beta_{n+1}\right) / 2, \mu_{1}=\bar{\beta} e^{i \theta / 2}, \mu_{2}=K \bar{\beta} e^{i \theta / 2}$. The circle does not pass through any poles of the integrand. From (2.17),

$$
\begin{gathered}
\Delta=c_{1} \mu_{1}^{2}\left[\cosh (m+1) \mu_{1} a+\cosh (m-1) \mu_{1} a\right]+c_{2} \mu_{1}^{2} \sinh \mu_{1} a \\
+\mu_{1}\left[c_{3} \sinh (m+1) \mu_{1} a+c_{4} \sinh (m-1) \mu_{1} a\right]+c_{5} \mu_{1} \cosh \mu_{1} a \\
+c_{6}\left[\cosh (m+1) \mu_{1} a-\cosh (m-1) \mu_{1} a\right]+c_{7} \sin \mu_{1} a \\
|\Delta|=\frac{1}{2} \mid \mu_{1}^{2} \exp \left[(m+1) \mu_{1} a\right]\left\{c_{1}+c_{1} \exp \left[-(m+1) \mu_{1} a\right]+c_{1} \exp \left(-2 \mu_{1} a\right)+\cdots\right. \\
+\frac{1}{\mu_{1}}\left[c_{2}-c_{2} \exp \left\{-2(m+1) \mu_{1} a\right\}+c_{3} \exp \left(-2 \mu_{1} a\right)+\cdots\right] \\
\left.\quad+\frac{1}{\mu_{1}^{2}}\left[c_{4}+c_{4} \exp \left\{-2(m+1) \mu_{1} a\right\}+c_{4} \exp \left(-2 \mu_{1} a\right)+\cdots\right]\right\} \\
\geq \frac{1}{2} \bar{\beta}^{2} \exp \left[(m+1) \bar{\beta} a \cos \frac{1}{2} \theta\right]\left\{-\left|c_{1}\right|+\cdots\right. \\
\left.\quad+\frac{1}{\bar{\beta}}\left[-2\left|c_{2}\right|-\left|c_{3}\right|+\cdots\right]+\frac{1}{\bar{\beta}^{2}}\left[-\left|c_{4}\right|+\ldots\right]\right\} .
\end{gathered}
$$

Here $m=K(b / a-1), c_{1}, c_{2}, \ldots, c_{7}$ are constants independent of $R$ and $\theta$, and we used $|x-y| \geq|x|-|y|$. With this,

$$
|\Delta|>C_{1} \bar{\beta}^{2} \exp \left[(m+1) \bar{\beta} a \cos \frac{1}{2} \theta\right]
$$

where $C_{1}$ is constant for fixed $\bar{\beta}$. The terms in the numerator are also reduced similarly. In this case we use $\sinh \mu_{1} a \leq \cosh \mu_{1} a$ and $|x+y| \leq|x|+|y|$. For convenience, we use partial fractions and consider each term separately. We note further that (cf. (2.14))

$$
a \chi_{i}^{\prime}(a)-\chi_{i}(a)=\int^{a} r^{\prime}\left[b_{i}-f_{i}\left(r^{\prime}\right)\right] \psi_{i}\left(r^{\prime}\right) d r^{\prime}
$$

and that $\left|\int f(x) \phi(x) d x\right| \leq M \int|\phi(x)| d x$ if $|f(x)| \leq M$ [13, p. 324]. We then verify that with $\xi=(m+1)(a-r) s_{1}^{-1 / 2}, 0 \leq \xi \leq(m+1) a s_{1}^{-1 / 2},\left|\Delta_{1}^{\prime} / \Delta\right|$ satisfies conditions of the theorem as $R$ (i.e. $n) \rightarrow \infty$. The second integral is also treated the same way. The analysis is long, but similar to one given by Carslaw and Jaeger [6, p. 399].

2.5. Constant initial temperatures. We assume that $f_{1}=f_{2}=f=$ constant and that everything else is the same as before. A straightforward reduction from (2.18)-(2.19) to (2.29)-(2.30) below is possible because of the choice of $\chi_{i}$ we made in (2.14). (Otherwise, the coefficients $B, C, D$ will have to be freshly calculated.) The coefficient matrix in (2.15) and the determinant $\Delta$ are unchanged, and

$$
R_{1}=R_{2}=0, \quad R_{3}=-\left(b_{2}-f\right) b B i .
$$


As before, the only singularities are the simple poles located at $\lambda_{n}=-\left(s_{1} / a^{2}\right) \beta_{n}^{2}$, $n=0,1,2, \ldots$. For the integration we take a Bromwich contour similar to Fig. 1. Alternatively, from the general solutions $(2.25)-(2.26)$ we obtain

$$
\left.\begin{array}{rl}
T_{1}(r, t)=b_{1} & +\frac{2 \sigma b B i\left(f-b_{2}\right)}{r} \sum_{n=1}^{\infty} \frac{\beta_{n} \sin \beta_{n} \sin m \beta_{n}}{\Phi\left(\beta_{n}\right)} \sin \left(\frac{\beta_{n} r}{a}\right) \exp \left(-F o \beta_{n}^{2}\right), \\
T_{2}(r, t)=b_{2} & +\frac{2 b B i\left(f-b_{2}\right)}{r} \sum_{n=1}^{\infty} \frac{\beta_{n} \sin ^{2} \beta_{n} \sin m \beta_{n}}{\Phi\left(\beta_{n}\right)} \sin \left(K \frac{r-a}{a} \beta_{n}\right) \\
& \times\left[\sigma \cot \left(K \frac{r-a}{a} \beta_{n}\right)+\cot \beta_{n}+\frac{\zeta}{\beta_{n}}\right] \exp \left(-F o \beta_{n}^{2}\right) .
\end{array}\right\}
$$

Here $b_{1}=b_{2}=T_{\infty}+q_{b} / h_{2}$, and $\beta_{n}>0$ are the roots of (2.20). The expression for $\Phi\left(\beta_{n}\right)$ was given in (2.27).

\section{Recovery of the temperature condition, mixed boundary, and related solutions.}

3.1. Composite spherical shells. Temperature specified at the outer surface. Let $T=T_{b}$ on $r=b, t>0$. By taking the limit $B i \rightarrow \infty$ (cf. Eqs. (2.25)-(2.27)) the solution for the temperature boundary condition is obtained. The results will not be shown here. The poles are the roots of $\lim _{B i \rightarrow \infty}(\Delta / B i)=0$, i.e. (2.23).

As a further simplification let the initial temperatures be constant, $f_{1}=f_{2}=f$. Then

$$
\frac{1}{\beta_{n}} \lim _{B i \rightarrow \infty} \frac{\Phi\left(\beta_{n}\right)}{B i}=\sigma m \beta_{n} \sin ^{2} \beta_{n}+\beta_{n} \sin ^{2} m \beta_{n}+\frac{\zeta}{\beta_{n}} \sin ^{2} \beta_{n} \sin ^{2} m \beta_{n} \equiv \Omega\left(\beta_{n}\right) .
$$

After some algebra, for $\beta_{n}>0$ the solution reduces to

$$
\left.\begin{array}{l}
T_{1}(r, t)=T_{b}+\frac{2 b \sigma\left(f-T_{b}\right)}{r} \sum_{n=1}^{\infty} \frac{\sin \beta_{n} \sin m \beta_{n}}{\Omega\left(\beta_{n}\right)} \sin \left(\frac{\beta_{n} r}{a}\right) \exp \left(-F_{o} \beta_{n}^{2}\right), \\
T_{2}(r, t)=T_{b}+\frac{2 b \sigma\left(f-T_{b}\right)}{r} \sum_{n=1}^{\infty} \frac{\sin ^{2} \beta_{n}}{\Omega\left(\beta_{n}\right)} \sin \left(K \frac{b-r}{a} \beta_{n}\right) \exp \left(-F_{o} \beta_{n}^{2}\right) .
\end{array}\right\}
$$

If we set $T_{b}=0, f=V$, we recover Carslaw and Jaeger's solution [7, p. 351].

3.2. Solid sphere, heat flux at surface, radiation to medium at $T_{\infty}$. We assume the sphere is receiving the heat flux $q$ at the surface and that its initial temperature is $T_{i}(r)$. Let $B i=h a / \kappa$ be the Biot number. Dropping all subscripts, the solution to (2.1) is

$$
\begin{gathered}
T(r, t)=b_{0}+\frac{2}{a r} \sum_{n=1}^{\infty} \frac{(B i-1)^{2}+\beta_{n}^{2}}{B i^{2}-B i+\beta_{n}^{2}} I\left(\beta_{n}\right) \sin \left(\frac{\beta_{n} r}{a}\right) \exp \left[-F o \beta_{n}^{2}\right], \\
I\left(\beta_{n}\right)=\int_{0}^{a}\left[T_{i}(r)-b_{0}\right] \sin \left(\frac{\beta_{n} r}{a}\right) r d r .
\end{gathered}
$$

Here $b_{0}=T_{\infty}+q / h, F o=s t / a^{2}$, and $\beta_{n}$ are the positive roots of $\beta \cot \beta=-(B i-1)$. Since $B i \geq 0$, the roots are real and distinct. From Theorem $2, \S 4.1, \beta \neq 0$ when $B i=1$, and thus $\lambda=0$ is not a pole. However if $B i=0$ and $q=0$, the inversion integral (not shown here) has a pole at $\lambda=0$. In this case, $b_{0}=0, s=1$, and the constant term 
$[f(0) / g(0)] / r=3 a^{-3} \int_{0}^{a} T_{i}(r) r^{2} d r$ will be added to (3.3); cf. (2.21). This term may not be derived from (3.3) as $\beta=0$ is not part of the set. If $B i=0$ but $q \neq 0$, see [3], $[$. If the surface temperature is specified, take $B i \rightarrow \infty$ and $\beta_{n}=n \pi$. For time-dependent heat flux or time-dependent boundary temperature, Duhamel's theorem will be used (cf. $\S 5$, below).

3.3. Hollow sphere, heat flux at boundary, radiation to media at unequal temperatures. This is a generalization of Carslaw and Jaeger's Fourier series solution for the hollow sphere [7, pp. 246-247]. Let $q_{a}, T_{a}$ be the heat flux and radiation sink temperature for the surface at $r=a$, and $q_{b}, T_{b}$ the same for the surface at $r=b$. Let $T_{i}(r)$ be the initial temperature. The governing DE is (2.1) without the subscripts. The linearized BCs are

$$
\begin{array}{rlrlrl}
\kappa \frac{\partial T}{\partial r} & =h_{1}\left(T-T_{a}\right)-q_{a}, & r & =a, & t>0, \\
-\kappa \frac{\partial T}{\partial r} & =h_{2}\left(T-T_{b}\right)-q_{b}, & & r=b, & & t>0 .
\end{array}
$$

As before, we use the transformations $u=\operatorname{Tr}$ and $u=a_{0}+b_{0} r+v$. Let the Biot numbers be $B i_{1}=h_{1} a / \kappa, B i_{2}=h_{2} b / \kappa$. The constants $a_{0}, b_{0}$ will be chosen to make the $\mathrm{BCs}$ (in the transformed state) homogeneous. Thus, if $B i_{1} \times B i_{2} \neq 0$, then

$$
\begin{gathered}
a_{0}=a b\left(b-a+\frac{b}{B i_{1}}+\frac{a}{B i_{2}}\right)^{-1}\left(T_{a}-T_{b}+\frac{q_{a}}{h_{1}}-\frac{q_{b}}{h_{2}}\right), \\
b_{0}=T_{a}+\frac{q_{a}}{h_{1}}+b \frac{B i_{1}+1}{B i_{1}}\left(b-a+\frac{b}{B i_{1}}+\frac{a}{B i_{2}}\right)^{-1}\left(T_{b}-T_{a}+\frac{q_{b}}{h_{2}}-\frac{q_{a}}{h_{1}}\right) . \\
\text { If } B i_{1}=0 \text {, then } \quad a_{0}=\frac{q_{a} a^{2}}{\kappa} \text { and } b_{0}=T_{b}+\frac{q_{b}}{h_{2}}+\frac{B i_{2}-1}{B i_{2}} \frac{q_{a} a^{2}}{b \kappa} . \\
\text { If } B i_{2}=0 \text {, then } \quad a_{0}=-\frac{q_{b} b^{2}}{\kappa} \text { and } b_{0}=T_{a}+\frac{q_{a}}{h_{1}}+\frac{B i_{1}+1}{B i_{1}} \frac{q_{b} b^{2}}{a \kappa} .
\end{gathered}
$$

We take the Laplace transform of the equations and then show that the subsidiary equation has the solution

$$
\begin{gathered}
\bar{v}=C \cosh q r+D \sinh q r-\frac{1}{q s} \chi(r), \\
\chi(r)=\int_{r}^{b}\left[a_{0}+b_{0} r^{\prime}-T_{i}\left(r^{\prime}\right) r^{\prime}\right] \sinh q\left(r^{\prime}-r\right) d r^{\prime} .
\end{gathered}
$$

From the Inversion Theorem,

$$
v(r, t)=\frac{1}{2 \pi i} \int_{\gamma-i \infty}^{\gamma+i \infty} e^{\lambda t}\left[\frac{\Delta_{2}^{\prime} \cosh \mu r+\Delta_{3}^{\prime} \sinh \mu r}{\Delta}-\chi(r)\right] \frac{d \lambda}{\sqrt{(\lambda s)}} .
$$

Here

$$
\begin{aligned}
\Delta=\left[b\left(B i_{1}+1\right)\right. & \left.+a\left(B i_{2}-1\right)\right] \mu \cosh \mu(b-a) \\
& +\left[\left(B i_{1}+1\right)\left(B i_{2}-1\right)+\mu^{2} a b\right] \sinh \mu(b-a),
\end{aligned}
$$

and $\Delta_{2}^{\prime}, \Delta_{3}^{\prime}$ are similar algebraic expressions. The second term in (3.6) has no contribution to the integral. The only singularities of the first integrand are the poles at 
$\lambda_{n}=-s \beta_{n}^{2} /(b-a)^{2}, n=1,2,3, \ldots$ The poles are the positive roots of $\Delta=0$, i.e.,

$$
\beta \cot \beta=-\frac{(b-a)\left(B i_{1}+1\right)\left(B i_{2}-1\right)-\beta^{2} a b(b-a)^{-1}}{b\left(B i_{1}+1\right)+a\left(B i_{2}-1\right)}=-\left(L_{1}-L_{2} \beta^{2}\right) .
$$

Here $L_{1}, L_{2}$ are parametric constants. For the solution of this equation, see $\S 4.4$.

Evaluating the residues and summing over $n$, the solution is

$$
\begin{aligned}
T(r, t)=b_{0}+\frac{a_{0}}{r}+\frac{2}{r} \sum_{n=1}^{\infty} \frac{1}{\Phi\left(\beta_{n}\right)} & {\left[\left(\Delta_{2}^{\prime}\right)_{\lambda=\lambda_{n}} \cos \left(\frac{\beta_{n} r}{b-a}\right)\right.} \\
& \left.-\left(i \Delta_{3}^{\prime}\right)_{\lambda=\lambda_{n}} \sin \left(\frac{\beta_{n} r}{b-a}\right)\right] \exp \left[-\frac{s}{(b-a)^{2}} \beta_{n}^{2} t\right] .
\end{aligned}
$$

Here $i \Delta_{3}^{\prime}$ is real. With further reduction and simplification we obtain

$$
\begin{gathered}
T(r, t)=b_{0}+\frac{a_{0}}{r}+\frac{2}{r} \sum_{n=1}^{\infty} \frac{I\left(\beta_{n}\right)}{\Omega\left(\beta_{n}\right)}\left[\frac{b^{2} \beta_{n}^{2}}{(b-a)^{2}}+\left(B i_{2}-1\right)^{2}\right] \\
\times \Psi_{n}(r) \exp \left[-\frac{s}{(b-a)^{2}} \beta_{n}^{2} t\right],
\end{gathered}
$$

where

$$
\begin{aligned}
\Psi_{n}(r) & =\frac{a \beta_{n}}{b-a} \cos \left(\frac{r-a}{b-a} \beta_{n}\right)+\left(B i_{1}+1\right) \sin \left(\frac{r-a}{b-a} \beta_{n}\right), \\
\Omega\left(\beta_{n}\right) & =\left[b\left(B i_{1}+1\right)+a\left(B i_{2}-1\right)\right]\left[\left(B i_{1}+1\right)\left(B i_{2}-1\right)+\frac{a b \beta_{n}^{2}}{(b-a)^{2}}\right] \\
& +(b-a)\left[\left(B i_{1}+1\right)^{2}+\frac{a^{2} \beta_{n}^{2}}{(b-a)^{2}}\right]\left[\left(B i_{2}-1\right)^{2}+\frac{b^{2} \beta_{n}^{2}}{(b-a)^{2}}\right], \\
I\left(\beta_{n}\right) & =\int_{a}^{b}\left[T_{i}(r) r-b_{0} r-a_{0}\right] \Psi_{n}(r) d r .
\end{aligned}
$$

The passage from (3.9) to (3.10) involves considerable algebra, but a full reduction is not necessary from a computational perspective. If we set $q_{a}=q_{b}=0$, solution (3.10) will be in full agreement with the recent results of Noda et al. [17, p. 131], who obtained a solution by Fourier series.

Special cases. If $B i_{1}=B i_{2}=0$ but $q_{1} \neq 0, q_{2} \neq 0$, then the problem may not have a steady state solution; see [3], [8]. If $B i_{1}=B i_{2}=0$ and $q_{1}=q_{2}=0$, then $\lambda=0$ is also a pole; see this from (3.8) as $\beta \rightarrow 0$. In this case $a_{0}=b_{0}=0, s=1$, and the constant term $[f(0) / g(0)] / r=3(b-a)^{-3} \int_{a}^{b} T_{i}(r) r^{2} d r$ will be added to the solution above; cf. (2.21). The solution for the solid sphere is realized by setting $a=0, B i_{1}=0$. For mixed boundary conditions take the appropriate $B i$ limit. Thus if $B i_{2}=\infty$, the poles are the roots of $\beta \cot \beta=-\left(b a^{-1}-1\right)\left(B i_{1}+1\right)$. For its solution, see the next section. If the temperature is specified at both ends take $B i_{1} \rightarrow \infty, B i_{2} \rightarrow \infty$, and $\beta_{n}=n \pi$. 
4. Evaluating the roots. In the literature, even when numerical results are given, very little is said of the roots and their properties, or a systematic method given for their determination. Recently there has been a renewed interest in the closed-form solutions. Analytical solutions are being used as benchmarks for validation purposes. We begin with three primitive equations $\lambda \tan \lambda=C, \lambda \cot \lambda=-C$, and $\sigma \cot m \theta+\cot \theta=0$ in which $C, \sigma, m$ are parameters. Evidently, parameter $m$ controls frequency and $\sigma$ controls amplitude of the third equation. The first two equations are common in diffusion, vibration, and acoustics [9], 18, 7]. Full asymptotic solutions have been published only recently [8]. In the past there have been attempts to find the roots of $\lambda \tan \lambda=C, \lambda \cot \lambda=-C$, and $\tan \lambda=\left(B i_{1}+B i_{2}\right) \lambda /\left(\lambda^{2}-B i_{1} B i_{2}\right)$. Tables of roots have been compiled for use as apparatus; e.g., see [7, 9], 23, Tables 4.19, 4.20]. For hybrid-algorithmic methods, see [22, [12]. This author feels that in contradistinction to these the asymptotic solutions of $[8]$ are direct, concise, and more powerful. The third primitive equation appears in composite problems [7. We show that a solution of complex equations, e.g. (2.20), boils down to making an appropriate use of the primitive equations. Using the methods described here we calculated for each equation up to 1000 roots and found the method accurate and efficient.

In solving $\sigma \cot m \theta+\cot \theta=0$ or $\tan m \theta+\sigma \tan \theta=0$, it is necessary to bear in mind that both equations are derived from $\sigma \cos m \theta \sin \theta+\cos \theta \sin m \theta=0$, the left-hand side of which will be in the denominator of the inversion integrand. If $m$ is an integer or a rational fraction, then depending upon the choice, the common roots of $\sin m \theta=0$ and $\sin \theta=0$, or of $\cos m \theta=0$ and $\cos \theta=0$, will also be the poles. These poles give rise to additional series solutions, but are absent if $m$ is irrational or transcendental. In the examples below we shall not always point this out explicitly.

\subsection{Preliminaries.}

Lemma 1. Let $p, q$ be integers without a common factor. Then $\cos p \alpha=0$ and $\cos q \alpha=0$ have no roots in common if one of $p, q$ is even. If $p, q$ are both odd and $n$ is the set of nonnegative integers, then the common roots of $\cos p \alpha=0$ and $\cos q \alpha=0$ are $\alpha= \pm\left(n+\frac{1}{2}\right) \pi, n=0,1,2, \ldots$.

Corollary 1. If $m=p / q$, then $\cos m \theta=0$ and $\cos \theta=0$ have only one common root in $(0, q \pi]$.

Proof. It is sufficient to consider only the positive roots. Let $p \alpha=\left(l+\frac{1}{2}\right) \pi, q \alpha=$ $\left(r+\frac{1}{2}\right) \pi$, where $l, r=0,1,2, \ldots$ For $\cos p \alpha=0$ and $\cos q \alpha=0$ to have common roots we should have

$$
q-p=2(r p-l q) .
$$

If $p=q$, then $l=r$, a trivial solution. Now the right-hand side of the above equation being even, for equality $p$ and $q$ must both be odd. This proves the first part. With $p, q$ odd, we select $l$ for which

$$
r=(2 l+1) \frac{q}{2 p}-\frac{1}{2}, \quad l=0,1,2, \ldots
$$


is zero or a whole number. For these select values of $r, \alpha= \pm\left(r+\frac{1}{2}\right) \pi / q= \pm\left(l+\frac{1}{2}\right) \pi / p$. Since $2 r=q[(2 l+1) / p]-1$, it is evident that $2 l+1$ is an odd multiple of $p,(2 l+1) / p=$ $2 n+1$. Therefore the common roots are $\alpha= \pm\left(n+\frac{1}{2}\right) \pi, n=0,1,2, \ldots$.

LEMma 2. Let $p, q$ be integers without a common factor and let $n$ be the set of nonnegative integers. Then the common roots of $\sin p \alpha=0$ and $\sin q \alpha=0$ are $\alpha= \pm n \pi$, $n=0,1,2 \ldots$

LEMma 3. Let $p, q$ be integers without a common factor. Then $\sin p \alpha=0$ and $\cos q \alpha=0$ have no roots in common if $p$ is odd or $q$ is even. If $p$ is even and $n$ is the set of nonnegative integers, then the common roots of $\sin p \alpha=0$ and $\cos q \alpha=0$ are $\alpha= \pm\left(n+\frac{1}{2}\right) \pi$, $n=0,1,2, \ldots$.

Corollary 1. If $m=p / q$, then $\sin m \theta=0$ and $\cos \theta=0$ have only one common root in $(0, q \pi)$.

THEOREM 1. The roots of $\lambda \tan \lambda=C$ in the positive half-plane are approximately given by

$$
\lambda_{n}= \begin{cases}\lambda_{0} \approx \sqrt{C}\left(1-\frac{1}{3} C\right), n \pi+\tan ^{-1} \frac{C}{n \pi} & (C<\lambda), \\ (2 n+1) \frac{\pi}{2}-\tan ^{-1}\left[(2 n+1) \frac{\pi}{2 C}\right] & (C>\lambda),\end{cases}
$$

where $C$ is constant and $n=0,1,2, \ldots$.

Theorem 2. The roots of $\lambda \cot \lambda=-C$ in the positive half-plane are approximately given by

$$
\lambda_{n}= \begin{cases}(2 n+1) \frac{\pi}{2}+\tan ^{-1} \frac{2 C}{(2 n+1) \pi} & (C<\lambda), \\ (n+1) \pi-\tan ^{-1} \frac{(n+1) \pi}{C} & (C>\lambda),\end{cases}
$$

where $C$ is a constant $\geq-1$ and $n=0,1,2, \ldots$.

The roots are all real. For an asymptotic solution of these, see [8]. For $\lambda \cot \lambda=-C$, the lower bound for $C$ is to ensure the roots are real. In this case $\lambda_{0} \neq 0$ when $C=0$; however, as $C \rightarrow-1, \lambda_{0} \rightarrow 0$.

4.2. Roots of $\sigma \cot m \theta+\cot \theta=0$. The equation is written as

$$
\sin (m+1) \theta=\frac{\sigma-1}{\sigma+1} \sin (m-1) \theta .
$$

We first assume $m$ to be an integer or a rational fraction. The general case follows.

4.2.1. $m$ an integer or a rational fraction.

TheOREM 3. Let $m=p / q$ be a rational fraction, where $p, q$ are integers reduced in their lowest terms. Then the number of principal roots in $[0, q \pi)$ of $\tan m \theta+\sigma \tan \theta=0$ is $s=p+q-1$ or $s=p+q$ according as $p, q$ are both odd, or one is odd and the other even. All other roots can be found from the recurrence relation $\theta_{n}-\theta_{n-s}=q \pi, n>s$.

Corollary 1. The number of principal roots in $[0, q \pi)$ of $(4.3)$ is $s=p+q$. 
Proof. For $m=1$ or $\sigma=0,1, \infty$, the solution is trivial. Let $\theta=q \alpha$, so that $\tan p \alpha=-\sigma \tan q \alpha$. By expanding both sides we have

$$
\frac{p \tan \alpha-\left(\begin{array}{l}
p \\
3
\end{array}\right) \tan ^{3} \alpha+\left(\begin{array}{l}
p \\
5
\end{array}\right) \tan ^{5} \alpha-\cdots}{1-\left(\begin{array}{l}
p \\
2
\end{array}\right) \tan ^{2} \alpha+\left(\begin{array}{l}
p \\
4
\end{array}\right) \tan ^{4} \alpha-\cdots}=-\sigma \frac{q \tan \alpha-\left(\begin{array}{l}
q \\
3
\end{array}\right) \tan ^{3} \alpha+\left(\begin{array}{l}
q \\
5
\end{array}\right) \tan ^{5} \alpha-\cdots}{1-\left(\begin{array}{l}
q \\
2
\end{array}\right) \tan ^{2} \alpha+\left(\begin{array}{l}
q \\
4
\end{array}\right) \tan ^{4} \alpha-\cdots} .
$$

The last terms in the numerator and denominator of $\tan m \alpha$ are

$$
(-1)^{\frac{m-2}{2}} m \tan ^{m-1} \alpha \quad \text { and } \quad(-1)^{\frac{m}{2}} \tan ^{m} \alpha \quad(m \text { even })
$$

and

$$
(-1)^{\frac{m-1}{2}} \tan ^{m} \alpha \quad \text { and } \quad(-1)^{\frac{m-1}{2}} m \tan ^{m-1} \alpha \quad(m \text { odd }) .
$$

Proof is by induction. The substitution of $t=\tan \alpha$ reduces (4.4) to a linear algebraic equation with constant coefficients. For each root $t$ we call $\alpha=\tan ^{-1} t$ a principal root. The higher roots are given by $\alpha=n \pi+\tan ^{-1} t$. Because the polynomial coefficients have alternating signs there are no negative principal roots. When $p, q$ are both odd, then (4.4) is of order $p+q-1$ with $p+q-1$ roots. On the other hand, if one is odd and the other even, then (4.4) has $p+q$ roots. Finally, the higher roots satisfy the recurrence relation $\alpha_{n}-\alpha_{n-s}=\pi$, i.e. $\theta_{n}-\theta_{n-s}=q \pi, n>s$.

Proof of Corollary 1. From the trigonometric identity

$$
(\sigma+1) \sin (m+1) \theta-(\sigma-1) \sin (m-1) \theta=2 \cos m \theta \cos \theta(\tan m \theta+\sigma \tan \theta),
$$

it immediately follows that the roots of (4.3) are given by the roots of $\tan m \theta+\sigma \tan \theta=0$, together with the common roots of $\cos m \theta=0$ and $\cos \theta=0$. By Lemma 1, Corollary 1 , and Theorem 3, the number of roots in $[0, q \pi)$ is $s=p+q$.

From (4.4),

$$
\tan \left(\alpha_{1}+\alpha_{2}+\cdots+\alpha_{s}\right)=\frac{s_{1}-s_{3}+s_{5}-\cdots}{1-s_{2}+s_{4}-\cdots}=0,
$$

so that $\alpha_{1}+\alpha_{2}+\cdots+\alpha_{s}$ is a multiple of $\pi$. Some further properties of the principal roots of $\tan p \alpha+\sigma \tan q \alpha=0$ are

$$
\begin{gathered}
\sum_{i=2}^{p+q-1} \tan \alpha_{i}=0, \quad \prod_{i=2}^{p+q-1} \tan \alpha_{i}=(-1)^{\frac{p+q-2}{2} \frac{p+q \sigma}{q+p \sigma}} \quad(p, q \text { odd }), \\
\sum_{i=1}^{p+q} \tan \alpha_{i}=0, \quad \prod_{i=2}^{p+q} \tan \alpha_{i}= \begin{cases}(-1)^{\frac{p+q-1}{2}}(p+q \sigma) & (p \text { odd }, q \text { even }), \\
(-1)^{\frac{p+q-1}{2}} \frac{p+q \sigma}{\sigma} & (q \text { odd }, p \text { even }) .\end{cases}
\end{gathered}
$$

\subsubsection{The general case.}

Theorem 4. Let $\chi=\sin (m+1) \theta-[(\sigma-1) /(\sigma+1)] \sin (m-1) \theta, \chi_{1}=\sin (m+1) \theta$, $m \geq 0, \sigma \geq 0$. Then the zeros of $\chi$ lie between the stationary points of $\chi_{1}$. Between two consecutive stationary points of $\chi_{1}, \chi$ has one and only one zero. 
Proof. We consider only the positive roots. Evidently $-1 \leq(\sigma-1) /(\sigma+1) \leq 1$ and $-1 \leq(m-1) /(m+1) \leq 1$. Let $\chi_{2}=[(\sigma-1) /(\sigma+1)] \sin (m-1) \theta$. The zeros of $\chi$ are determined by the intersections between the functions $\chi_{1}$ and $\chi_{2}$. Both $\chi_{1}, \chi_{2}$ are bounded periodic functions, symmetric about $y=0$. There is no phase difference. Let $\theta_{1}=\left(n-\frac{1}{2}\right) \pi /(m+1), \theta_{2}=\left(n+\frac{1}{2}\right) \pi /(m+1), n=1,2,3, \ldots$ be two consecutive stationary points of $\chi_{1}$.

The intersections between $\chi_{1}$ and $\chi_{2}^{+}=+\sin (m-1) \theta$ are given by $\theta_{r}=\left(r-\frac{1}{2}\right) \pi / m$ and $\theta_{r}=r \pi, r=1,2,3, \ldots$. Let the $\theta_{r}$ 's be combined and sorted in ascending order of magnitudes and denote these by $\theta_{r}^{+}$. Similarly, the intersections between $\chi_{1}$ and $\chi_{2}^{-}=-\sin (m-1) \theta$ are given by $\theta_{r}=r \pi / m$ and $\theta_{r}=\left(r-\frac{1}{2}\right) \pi$. Let the $\theta_{r}$ 's be combined and sorted in ascending order of magnitudes and denote these by $\theta_{r}^{-}$. Then

$$
\begin{array}{ll}
\left(n-\frac{1}{2}\right) \frac{\pi}{m+1} \leq \theta_{n}^{+} \leq \theta_{n}^{-} \leq\left(n+\frac{1}{2}\right) \frac{\pi}{m+1} \quad\left(\theta_{n}^{+} \leq \theta_{n}^{-}\right), \\
\left(n-\frac{1}{2}\right) \frac{\pi}{m+1} \leq \theta_{n}^{-} \leq \theta_{n}^{+} \leq\left(n+\frac{1}{2}\right) \frac{\pi}{m+1} \quad\left(\theta_{n}^{+} \geq \theta_{n}^{-}\right) .
\end{array}
$$

The outer equalities are for certain combinations of $m, n$, and $r$. The inner equality holds for $m=1$, in which case $\theta_{n}^{+}$and $\theta_{n}^{-}$coincide. The intersections are bounded between $\theta_{1}, \theta_{2}$. This is true for all $\sigma \geq 0$ because $(\sigma-1)(\sigma+1)^{-1}$ is continuous and bounded between -1 and +1 . Finally, two consecutive extrema of $\chi_{1}$ are separated by $\pi /(m+1)$. Since $m \geq 0$ it is verified that if $\theta_{n}^{+}$is inside $\left(\theta_{1}, \theta_{2}\right)$, then $\theta_{n-1}^{+}$and $\theta_{n+1}^{+}$are outside the interval, and similarly for $\theta_{n}^{-}$.

The roots of (4.3) are now isolated. As an initial approximation we use $\theta=\frac{1}{2}\left(\theta_{1}+\theta_{2}\right)$ $=n \pi /(m+1)$ and iterate. When $m$ is an integer or a rational fraction only the principal roots are calculated. The sum and product properties of the roots (see §4.2.1) furnish useful checks.

The roots of $\tan m \theta+\sigma \tan \theta=0$ may be obtained from the roots of $\chi=\sin (m+1) \theta-$ $[(\sigma-1) /(\sigma+1)] \sin (m-1) \theta=0$ by removing the common roots of $\cos m \theta=0$ and $\cos \theta=0$ from the set; see Corollary 1 of Theorem 3. Similarly, the roots of $\sigma \cot m \theta+$ $\cot \theta=0$ are obtained from the roots of $\chi=0$ by removing the common roots of $\sin m \theta=0$ and $\sin \theta=0$.

4.3. Roots of $\sigma \tan m \theta-\cot \theta=0$. This will be written as

$$
\cos (m+1) \theta=\frac{\sigma-1}{\sigma+1} \cos (m-1) \theta .
$$

We now state

Theorem 5. Let $\psi=\cos (m+1) \theta-[(\sigma-1) /(\sigma+1)] \cos (m-1) \theta, \psi_{1}=\cos (m+1) \theta$, $m \geq 0, \sigma \geq 0$. Then the zeros of $\psi$ lie between the stationary points of $\psi_{1}$. Between two consecutive stationary points of $\psi_{1}, \psi$ has one and only one zero.

The proof is along the lines of Theorem 4. As before, we use $\theta=\left(n+\frac{1}{2}\right) \pi /(m+1)$ as the initial approximation.

4.4. Roots of the eigenvalue equations. The method outlined here calculates all roots simultaneously. The general procedure is to construct a starting sequence $\{\bar{\beta}\}, \overline{\beta_{n}}>0$. By considering various parametric limits, the original equation is broken into simpler 
equations. We next construct a composite $\{\bar{\beta}\}$ whose elements are distinct and in ascending order of magnitudes. It is necessary that there be no gaps in $\{\bar{\beta}\}$. For the types of eigen-equations encountered in this paper this has always been feasible. Iterative refinement was done by Newton's method with under-relaxation, $\beta_{\text {new }}=\beta_{\text {old }}-\varpi\left(f / f^{\prime}\right)$, $\varpi<1$. Here $f$ stands for the residual. Close attention must be paid to $\beta_{n}-\beta_{n-1}$. Occasionally, if some $\beta_{n}$ shows a jump or a double root is found, a bisection or Ridders' method is applied locally. A graph will be helpful. See [20, p. 446] in this connection.

Roots of (3.8). Let $L=\left(\left|L_{1}\right| / L_{2}\right)^{1 / 2}$. The initial $\{\bar{\beta}\}$ are constructed from

$$
\begin{array}{ll}
\bar{\beta} \cot \bar{\beta}=-L_{1} & (\bar{\beta}<L), \\
\bar{\beta} \tan \bar{\beta}=\frac{1}{L_{2}} & (\bar{\beta}>L),
\end{array}
$$

and the residual equation is $f \equiv L_{2} \beta \sin \beta-\cos \beta-L_{1} \sin \beta / \beta=0$. Convergence is rapid. Note that as $n \rightarrow \infty, \beta_{n}-\beta_{n-1} \rightarrow \pi$, so that the large roots are nearly separated by $\pi$.

Alternatively, let $\tan \gamma=\left(B i_{1}+1\right)(b-a) /(\beta a), \tan \delta=\left(B i_{2}-1\right)(b-a) /(\beta b)$. Then from (3.8),

$$
\tan \beta=\tan (\gamma+\delta),
$$

so that $\beta=n \pi+\gamma+\delta, n=0,1,2, \ldots$ With $\beta_{n} \approx \bar{\beta}_{n}=\left(n+\frac{1}{2}\right) \pi$, the desired solution is

$$
\beta_{n}=n \pi+\tan ^{-1}\left[\frac{b-a}{\bar{\beta}_{n} a}\left(B i_{1}+1\right)\right]+\tan ^{-1}\left[\frac{b-a}{\bar{\beta}_{n} b}\left(B i_{2}-1\right)\right] .
$$

This is a convergent sequence. An asymptotic solution will be apparent.

Roots of (2.23), (2.24). We use the residual equation

$$
f \equiv \sigma \sin \beta \cos m \beta+\cos \beta \sin m \beta+\frac{\zeta}{\beta} \sin \beta \sin m \beta=0,
$$

which, unlike (2.23), avoids discontinuous functions and the problem that a change in the sign of the residual is not a sufficient condition that a root has been isolated. The following limiting solutions will be noted:

$\sigma \rightarrow 0$

$$
\sin m \beta=0 \text { and } \beta \cot \beta=-\zeta .
$$

$\sigma \rightarrow \infty$

$$
\begin{array}{cl}
\sin \beta=0 \text { and } m \beta \cot m \beta=-\frac{m \zeta}{\sigma} & \left(0 \leq \beta<\frac{|\zeta|}{\sigma}\right), \\
\sin \beta=0 \text { and } \sigma \cot m \beta+\cot \beta=0 & \left(\frac{|\zeta|}{\sigma} \leq \beta \leq \infty\right) .
\end{array}
$$

In practice we used common roots of (4.9) for $\sigma \leq 0.1$, and of (4.10a-b) for $\sigma>0.1$. Roots of (2.24) are determined similarly.

Roots of (2.20), (2.22). For fixed $B i$, we have the following.

For Bi small, Bi $\rightarrow 0$,

$$
\sigma \tan m \beta-\cot \beta=\frac{\zeta}{\beta},
$$

together with the common roots of $\sin \beta=0$ and $\cos m \beta=0$.

For intermediate Bi, 
$\sigma \rightarrow 0$

$$
\begin{gathered}
\cos \beta=0 \text { and } \cos m \beta=0 \quad(\beta<|\zeta|), \\
\cos \beta=0 \text { and } m \beta \cot m \beta=-(B i-1)\left(1-\frac{a}{b}\right) \quad(\beta>|\zeta|) . \\
\sin \beta=0 \text { and } m \beta \tan m \beta=\left[B i+\left(1-\frac{\kappa_{1}}{\kappa_{2}}\right) \frac{b}{a}-1\right]\left(1-\frac{a}{b}\right) .
\end{gathered}
$$$$
\sigma \rightarrow \infty
$$

For Bi large, $B i \rightarrow \infty$,

$$
\sigma \cot m \beta+\cot \beta=-\frac{\zeta}{\beta},
$$

together with the common roots of $\sin \beta=0$ and $\sin m \beta=0$.

We have used (4.11) for $B i \leq 5$, (4.12a)-(4.12c) for $5<B i<500$, and (4.13) for $B i \geq 500$. The division is arbitrary and there are overlaps. It is difficult to give a robust starting solution because there is no simple rule to bracket the roots. We overcame the difficulty by quasi-linearizing Newton's equation, which yields

$$
\beta_{\text {new }}=\beta_{\text {old }}-\delta \beta\left[\frac{f}{f^{\prime}}\right]_{\text {old }} \quad\left(f f^{\prime \prime} \neq 2 f^{\prime 2}\right),
$$

where $\delta \beta=2 f^{\prime 2} /\left(2 f^{\prime 2}-f f^{\prime \prime}\right)$. If $f f^{\prime \prime}=2 f^{\prime 2}$, then $\delta \beta \approx\left(-6 f / f^{\prime \prime \prime}\right)^{1 / 3}$.

5. Practical applications. Spacecraft propulsion tanks are usually spherical, made of titanium, and have heater strips on the surface to keep their fuel (typically anhydrous hydrazine $\mathrm{N}_{2} \mathrm{H}_{4}$, a liquid) above its freezing point $\left(2{ }^{\circ} \mathrm{C}\right)$. A temperature sensor is installed on the tank. There is no sensor for the liquid. The problem is to calculate the mass of fuel in the tank from a knowledge of the tank's temperature response. This determination is of considerable importance when only a small amount of fuel is left. Problems of this nature truly belong to inverse heat transfer problems (IHTP) because the uniqueness and sensitivity of the solution are not fully established. Nevertheless, some accepted IHTP examples are the determination of thermal conductivity and specific heat, the surface heat flux condition from the temperature history interior to a body, etc. [2], [19].

Experience has shown that the fuel mass is best determined by simulating the period when there is no mass transfer across boundaries and the tank temperature has a strong rising and falling profile. This happens when the heaters turn on and the tank temperature shows a steady rise until when the heating is turned off by a heater control switch. During cool down (heater off) the temperature drops relatively slowly.

Exact solution of the direct problem. Using the tank's internal wicking structure the liquid collects at the wall. A full discussion is outside the scope of this work. Here we simulate a spherical tank (region 2) with pressurized gaseous helium as the core (region 1). A thin liquid layer is assumed to be uniformly distributed on the inside wall of the tank. The liquid mass is lumped with the tank. We note that propulsion tanks are fully enclosed inside the spacecraft, and therefore tanks do not have a view to space. In the model, $T_{\infty}$ represents the inside structure temperature, and $T_{2}-T_{\infty}$ is small compared 
to $T_{\infty}$. With this, the linearization condition is satisfied. Let the initial temperatures be $T_{1}=T_{2}=T_{0}$. Substituting $\vartheta_{i}=T_{i}-T_{0}$ in $(2.1)-(2.6)$, the only nonhomogeneity is in the $\mathrm{BC}$ at $r=b$,

$$
-\kappa_{2} \frac{\partial \vartheta_{2}}{\partial r}=\left(h_{2}+h_{l}\right) \vartheta_{2}-F(t), \quad r=b, t>0
$$

where $F(t)=q(t)-h_{2}\left(T_{0}-T_{\infty}\right)-h_{l}\left(T_{0}-T_{s}\right)$, and $h_{l}$ is the heat transfer coefficient for the conduction heat loss through the tank's support points at temperature $T_{s}$.

In the present example,

$$
q(t)= \begin{cases}Q & \left(0<t<\tau_{1}\right), \\ 0 & \left(\tau_{1}<t<\tau_{2}\right), \\ Q & \left(\tau_{2}<t<\tau_{3}\right), \\ \vdots & \end{cases}
$$

The 'on/off' times are available from the spacecraft's telemetry. The constant heat input $Q$ is calculated from the ohmic resistance and applied voltage. All other parameters are known. For the example presented in Fig. 2(a), we took $a=0.247 \mathrm{~m}, b=0.25 \mathrm{~m}$, $T_{0}=T_{\infty}=T_{s}=15^{\circ} \mathrm{C}, h_{l}=0.127 \mathrm{Wm}^{-2} \mathrm{~K}^{-1}, \epsilon_{2}=0.1, Q=7.0 \mathrm{Wm}^{-2}$, residual fuel mass $1 \mathrm{~kg}, \tau_{j}=164,580,720,1128, \ldots \mathrm{min}$. The helium is at $0.689 \mathrm{MPa}$. The following were calculated: $K=2.024, \zeta=131.528, m=0.025, \sigma=268.214, B i=0.008$, $h_{2}=0.542 \mathrm{Wm}^{-2} \mathrm{~K}^{-1}$. We now set $F(t)=1$ and let $\Psi_{i}(r, t)$ be the auxiliary solution. It is verified that the $\Psi_{i}(r, t)$ will be given by $(2.29)-(2.30)$ by setting $f=0$ and $b_{1}=$ $b_{2}=\left(h_{2}+h_{l}\right)^{-1}$. By Duhamel's theorem [18, [7, 3], 8], the solution when $F(t)$ varies with time is

$$
\vartheta_{i}(r, t)=\frac{\partial}{\partial t} \int_{0}^{t} F(\tau) \Psi_{i}(r, t-\tau) d \tau=-\int_{0}^{t} F(\tau) \frac{\partial \Psi_{i}(r, t-\tau)}{\partial \tau} d \tau .
$$

Recognizing that $F(t)$ has jumps, the desired solution is

$$
\vartheta_{i}(r, t)= \begin{cases}Q \Psi_{i}(r, t) & \left(0<t<\tau_{1}\right), \\ Q\left[\Psi_{i}(r, t)-\Psi_{i}\left(r, t-\tau_{1}\right)\right] & \left(\tau_{1}<t<\tau_{2}\right) \\ \vdots & \end{cases}
$$

For heating and cooling with many discontinuities, Duhamel's theorem in Stieltjes integral form is

$$
\vartheta_{i}(r, t)=\int_{\tau=0}^{t} \Psi_{i}(r, t-\tau) \frac{d F(\tau)}{d \tau} d \tau+\sum_{j=0}^{n-1} \Psi_{i}\left(r, t-\tau_{j}\right) \Delta F_{j},
$$

where $\tau_{j}$ is the time at which a step change of $\Delta F_{j}$ (with signs) occurs.

5.1. An error metric. Let $\varphi$ represent the observed tank temperature and let $T$ be the exact solution when the fuel mass is $m_{f}$. We define the metric $D[\varphi, T]=\|\varphi-T\|$, where $\|f\|=\sqrt{(f, f)}$ is the norm and $(f, g)$ is the scalar or inner product of $f$ and $g$. In 


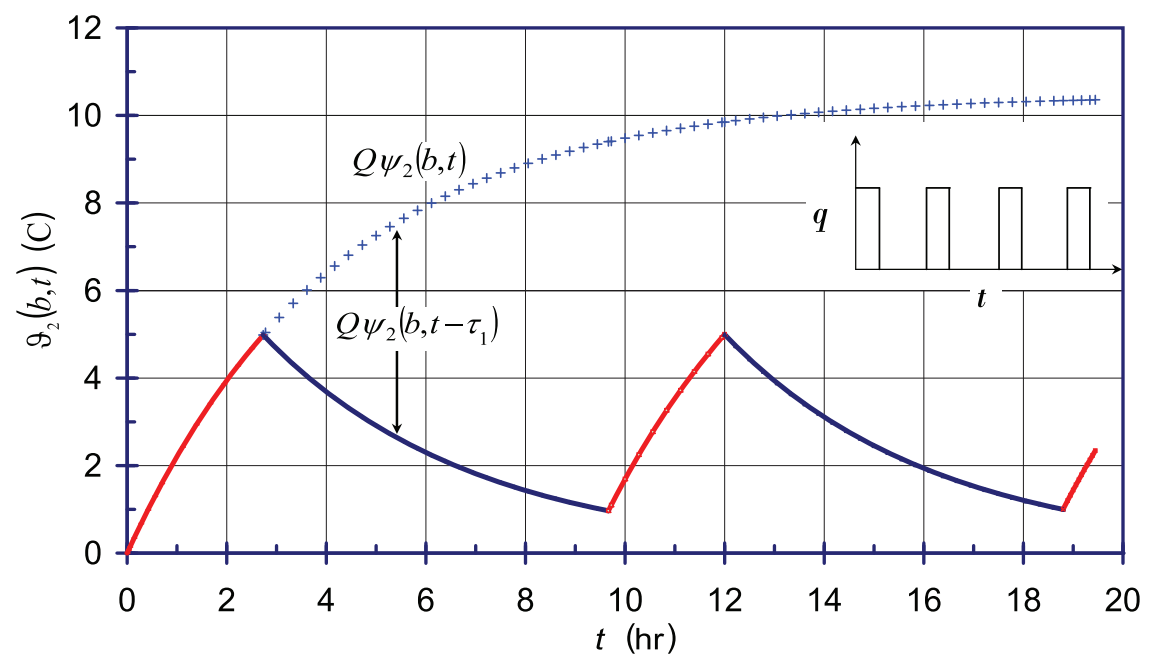

FIG. 2(a). Surface temperature of a two-layer propulsion tank due to heating and cooling. $\vartheta_{2}=T_{2}-T_{0}$. Residual fuel mass $=1 \mathrm{~kg}$.

the interval $\left(t_{1}, t_{2}\right)$ and $L^{2}$,

$$
D[\varphi, T]=\left[\int_{t_{1}}^{t_{2}}(\varphi-T)^{2} d t\right]^{1 / 2}
$$

With $\|\varphi\|^{2}=\int_{t_{1}}^{t_{2}} \varphi^{2} d t>0,\|T\|^{2}=\int_{t_{1}}^{t_{2}} T^{2} d t>0$, we define the error metric $N$ as

$$
N^{2}=\frac{\|\varphi-T\|^{2}}{\|\varphi\|^{2}} \text {. }
$$

Evidently, $N \rightarrow 0$ as $T \rightarrow \varphi$. If for some positive $\varepsilon, N<\varepsilon$, then $T$ is said to have converged in the mean [1]. In $\ell_{2}$ the error metric is

$$
N^{2}=\frac{\sum_{i=1}^{n}\left(\varphi_{i}-T_{i}\right)^{2}}{\sum_{i=1}^{n} \varphi_{i}^{2}} .
$$

If $N \rightarrow 0$ as $n \rightarrow \infty$ we say that an exact solution has been realized. The relative error

$$
\left[\sum_{i=1}^{n}\left(\rho c_{p} \varphi_{i}-\rho c_{p} T_{i}\right)^{2}\right]^{1 / 2} /\left[\sum_{i=1}^{n}\left(\rho c_{p} \varphi_{i}\right)^{2}\right]^{1 / 2}
$$

has a thermodynamic meaning: it is the ratio of the unaccounted heat to the total energy content. We find the fuel mass with the least error and call it the nominal prediction. Fig. 2(b) illustrates the ideas. The Levenberg-Marquardt method may also be used to minimize the error. For confidence bounds, we take $\varphi_{\text {measured }}=T_{\text {exact }}+\alpha \sigma$, where $\sigma$ is the standard deviation of observed data, and $\alpha$ (with signs) is calculated randomly for some assumed bounds. Using statistical analysis we then assign an uncertainty bar (confidence levels) to the prediction to account for the uncertainty in the observed temperature data $\left( \pm 0.5^{\circ} \mathrm{C}\right.$ or less $)$. 


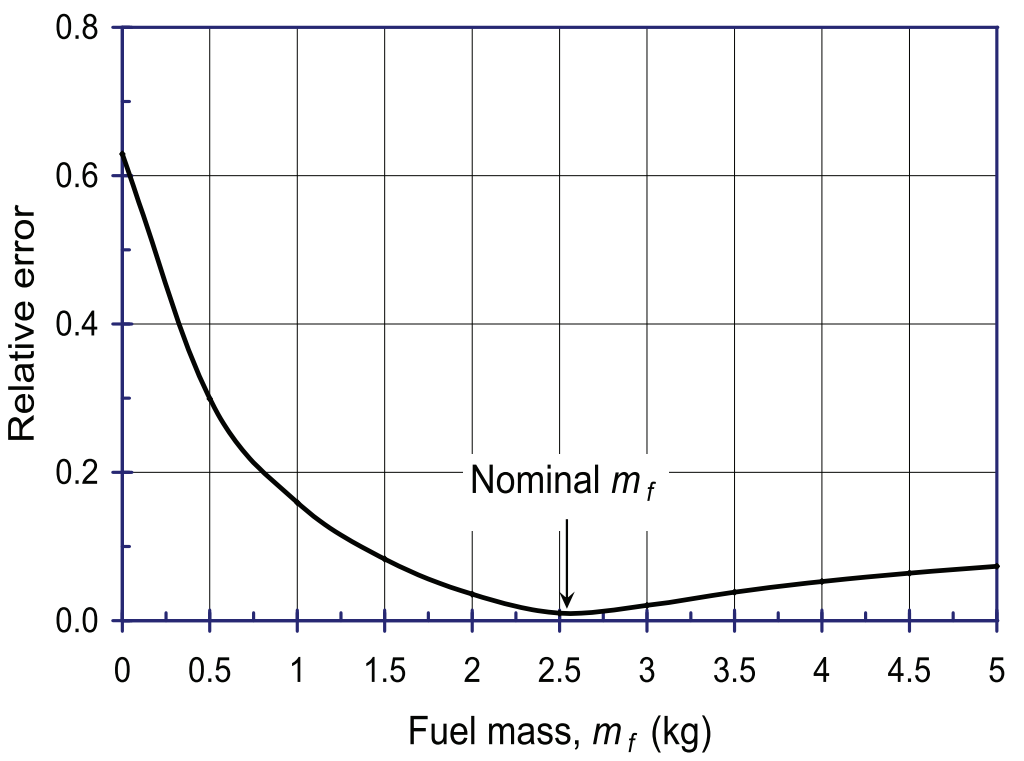

FIG. 2(b). Error vs. fuel mass.

6. Conclusions. The transient temperature response of composite spherical bodies is given in closed form for mixed and time-dependent boundary conditions. Solutions are obtained in infinite series. Simple methods were developed to calculate the roots of certain transcendental equations. The method and the solutions should find use in other branches of mechanics such as the thermoelastic response or creep of spherical shells, diffusion and evaporation in chemical processes, and cooling of glass in industry. For an application in another direction, the composite solution is used to calculate the residual fuel mass of an orbiting spacecraft. A measure of the uncertainty in the calculation is also given. Currently spacecraft do not have the means to relay back the status of their residual fuel. This makes the analytical determination extremely valuable in the planning and operation of aging spacecraft.

Acknowledgment. The author wishes to acknowledge journal reviewers for their insightful comments.

\section{REFERENCES}

[1] N. I. Akhiezer and I. M. Glazman, Theory of Linear Operators in Hilbert Space (translated by M. Nestell) 3rd printing, Frederick Ungar, New York, 1978. MR509335 (82g:47001b)

[2] J. V. Beck, B. Blackwell, and C. R. St. Clair, Jr., Inverse Heat Conduction: Ill-Posed Problems, Wiley-Interscience, New York, 1985.

[3] B. A. Boley and J. H. Weiner, Theory of Thermal Stresses, Dover reprint, New York, 1977.

[4] T. J. I'a. Bromwich, Symbolic methods in the theory of conduction of heat, Proc. Cambridge Phil. Soc., 20, 1921, 411-427. Here Bromwich devises a contour and method that now bears his name. Bromwich's and Carslaw's [5] papers were read the same day (2 May 1921) at Society's meeting. 
[5] H. S. Carslaw, The cooling of a solid sphere with a concentric core of a different material, Proc. Cambridge Phil. Soc., 20, 1921, 399-410. The Tripos problem mentioned in the Introduction is reproduced in this paper.

[6] H. S. Carslaw and J. C. Jaeger, A problem in conduction of heat, Proc. Cambridge Phil. Soc., 35, 1939, 394-404. MR0000456 (1:77a)

[7] H. S. Carslaw and J. C. Jaeger, Conduction of Heat in Solids (reprint of the 2nd edition), Clarendon Press, Oxford, 1973. MR0022294 (9:188a)

[8] B. K. Choudhury, Transient temperature and elastic response of a space-based mirror in the radiation-conduction environment, Quart. Appl. Math., 64 (2), 2006, 201-228. The order term in Eq. (2.3) of this paper strictly is $O\left[\left(T-T_{1}\right) / T_{1}\right]+O\left[\left(T-T_{1}\right) /\left(T_{1}+T_{2}\right)\right]$. MR2243860 (2007b:74021)

[9] J. Crank, The Mathematics of Diffusion (reprint of the 2nd edition), Clarendon Press, Oxford, 1994. The hollow sphere solution is misprinted. Thus, in Eq. (6.56), p. 101, $R_{n}(r)$ appears incorrectly in the argument of the exponential term. MR0082827(18:616c)

[10] W. A. Day, Heat Conduction within Linear Thermoelasticity, Springer Tracts in Natural Philosophy, vol. 30, Springer-Verlag, Berlin, 1985. MR804043 (87c:73001)

[11] G. M. L. Gladwell and J. R. Barber, Thermoelastic contact problems with radiation boundary conditions, Quart. J. Mech. Appl. Math., 36 (3), 1983, 403-407. MR714309 (85d:73005)

[12] A. Haji-Sheikh and J. V. Beck, An efficient method of computing eigenvalues in heat conduction, Numerical Heat Transfer B 38, 2000, 133-156.

[13] G. H. Hardy, A Course of Pure Mathematics (8th edition), Cambridge University Press, London, 1941.

[14] T. Hata, Transient thermal and residual stresses caused by creep in a sphere, in Theoretical and Applied Mechanics, vol. 22, Proc. 22nd Japan National Congress, University of Tokyo Press, Tokyo, 1972, 235-244.

[15] P. K. Jain, S. Singh, and Rizwan-uddin, Analytical solution to transient asymmetric heat conduction in a multilayer annulus, J. Heat Transfer, 131, 2009, 011304 (7 pages).

[16] N. W. McLachlan, Complex Variable Theory and Transform Calculus (2nd edition), Cambridge University Press, London, 1953. MR0059397 (15:524a)

[17] N. Noda, R. B. Hetnarski, and Y. Tanigawa, Thermal Stresses (2nd edition), Taylor and Francis, London, 2003.

[18] M. N. Özişik, Heat Conduction (2nd edition), Wiley-Interscience, New York, 1993.

[19] M. N. Özişik and H. R. B. Orlande, Inverse Heat Transfer: Fundamentals and Applications, Taylor and Francis, London, 2000.

[20] W. H. Press, S. A. Teukolsky, W. T. Vetterling, and B. P. Flannery, Numerical Recipes: The Art of Scientific Computing (3rd edition), Cambridge University Press, Cambridge, 2007. MR2371990 (2009b:65001)

[21] S. Singh, P. K. Jain, and Rizwan-uddin, Analytical solution to transient heat conduction in polar coordinates with multiple layers in radial direction, Int. J. Therm. Sciences, 47, 2008, 261-273.

[22] J. W. Stevens and R. Luck, Explicit approximations for all eigenvalues of the 1-D transient heat conduction equations, Heat Transfer Engineering, 20, 1999, 35-41.

[23] R. Zucker, Elementary transcendental functions, in M. Abramowitz and I. A. Stegun (eds.), Handbook of Mathematical Functions with formulas, graphs, and mathematical tables, Tables 4.19, 4.20, Dover, New York, 1970. MR0167642 (29:4914) 